\title{
Influência do suporte organizacional nas vivências de prazer e sofrimento no trabalho em um contexto hospitalar
}

\author{
Manueli Tomasi ${ }^{1, *}$, Vanessa Rissi ${ }^{2}$, \\ Jandir Pauli
}

${ }^{1}$ http://orcid.org/0000-0002-9117-1694 / Universidade do Vale do Rio dos Sinos (UNISINOS), Brasil

${ }^{2}$ http://orcid.org/0000-0002-1895-9354 / Faculdade IMED, Brasil

${ }^{3}$ http://orcid.org/0000-0003-4618-6958 / Faculdade IMED, Brasil

Resumo

O objetivo deste estudo é analisar a influência do suporte organizacional na ocorrência de vivências de prazer e sofrimento no trabalho inserido em um contexto hospitalar. Para tanto, foi realizada uma pesquisa investigativa quantitativa com cento e setenta e dois profissionais de um hospital privado no norte do Rio Grande do Sul. Os resultados mostraram que o suporte material para um melhor desempenho influencia significativamente nas vivências de prazer e estímulo no trabalho, enquanto a sobrecarga de trabalho e a precariedade da gestão do desempenho estão relacionadas com as vivências de sofrimento.

Palavras-chave: prazer e sofrimento, suporte organizacional, trabalho, hospital.

Influence of organizational support on the experiences of pleasure and suffering at work in a hospital context

Abstract

The aim of this study is to analyze the influence of organizational support on the occurrence of experiences of pleasure and suffering at work in a hospital context. For this, quantitative research was carried out with 172 professionals from a private hospital in the north of Rio Grande do Sul. The results showed that the material support for better performance significantly influences the experiences of pleasure at work, while work overload and poor performance management are related to the experiences of suffering.

Keywords: pleasure and suffering, organizational support, job, hospital.
Influencia del soporte organizacional en las vivencias de placer y sufrimiento en el trabajo en un contexto hospitalario

Resumen

El objetivo de este estudio es analizarla influencia del apoyo organizacional en la ocurrencia de experiencias de placer y sufrimiento en el trabajo insertado en un contexto hospitalario. Para tanto, se realizó una pesquisa investigativa cuantitativa con ciento setenta y dos profesionales de un hospital privado en el norte de Rio Grande do Sul. Los resultados mostraron que el apoyo material para un mejor rendimiento influye significativamente en las experiencias de placer y estimulo en el trabajo, mientras que la sobrecarga de trabajo y la precariedad de la gestión del desempeño están relacionadas con las experiencias de sufrimiento.
Palabras-clave: placer y sufrimiento, soporte organizacional, trabajar, hospital. 
Nas instituições de saúde, dentre elas os hospitais, o trabalho é dinâmico e considerado complexo devido ao tipo de serviço oferecido aos usuários e, ainda, por congregar uma diversidade de categorias profissionais para o seu adequado funcionamento (Brito, Santos, Soares, \& Camelo, 2015; Costa, Borges, \& Barros, 2015; Penaforte \& Araújo, 2016; Šimunić \& Gregov, 2012). Em adição, o contexto hospitalar envolve aspectos simbólicos como saúde, doença, vida e morte, exigindo alta demanda física e psicológica dos trabalhadores (Costa et al., 2015; Brito et al., 2015; Penaforte \& Araújo, 2016; Šimunić \& Gregov, 2012).

Assim considerado, o trabalho diário, as condições do ambiente, a estrutura e as funções no ambiente hospitalar, podem impactar negativamente no funcionamento organizacional, especialmente quando resultam em sobrecarga de trabalho, patologias laborais e absenteísmo (Camelo, 2011; Nogueira et al., 2018; Sangy, 2017). Não obstante, pesquisadores têm investido na análise dos aspectos subjetivos do trabalho em contexto hospitalar, para compreender, entre outras razões, quais elementos devem ser suportados pela organização do trabalho, para que se obtenha um contexto de trabalho menos nocivo à saúde dos trabalhadores e, ainda, produtivo (Bertoncello, Sousa, Marques, \& Oliveira, 2017).

Em atenção à literatura nacional e internacional, notabilizou-se o predomínio destas pesquisas a partir do ponto de vista dos profissionais com formação na área da saúde, como técnicos de enfermagem, enfermeiros e médicos (Brito et al., 2015; Costa et al., 2015; Penaforte \& Araújo, 2016; Rodrigues \& Faiad, 2019; Šimunić \& Gregov, 2012). Entretanto, é preciso que sejam consideradas as percepções das diferentes categorias profissionais envolvidas no objetivo institucional de prestar cuidados à saúde (Camelo, 2011; Nogueira et al., 2018; Sangy, 2017). Destarte, esta ampliação permitiria alinhar sistemicamente as expectativas dos trabalhadores com as da organização hospitalar (Costa et al., 2015).

Para tanto, os fatores subjetivos do trabalho representam o foco destes estudos, ao mesmo tempo em que atravessam os discursos do mundo do trabalho contemporâneo (Bendassolli, 2007; Bendassolli, 2012; Carmo et al., 2017; Dejours, 2008; Fleury, Formiga, de Souza, \& de Souza, 2017; Giongo, Monteiro, \& Sobrosa, 2015). Uma das abordagens que tem contribuído para este tipo de análise é a Psicodinâmica do Trabalho (Giongo et al., 2015; Flores \& Moura, 2018).

Nesta perspectiva teórica e metodológica, o trabalho ocupa um lugar central na construção da identidade, produzindo significados psíquicos, em que também se constroem relações sociais (Dejours, 2015; Freitas, Augusto, \& Mendes; Martins, Robazzi, \& Bobroff, 2010). São diversas as formas pelas quais o trabalhador percebe e vivencia as relações psicoafetivas com o trabalho que, por sua vez, são influenciadas pelos padrões relacionais estabelecidos entre o trabalhador e o seu trabalho, bem como pela convivência do indivíduo com a família, consigo mesmo, e com os outros (Carmo et al., 2017; Dejours, 1999; Lancman \& Sznelwar, 2004; Oleto, Melo, \& Lopes, 2013; Prestes et al. 2010).

Dependendo da psicodinâmica das variáveis, o trabalho pode ser percebido como fonte de prazer, atribuindo sentido à vida, elevando o status, incentivando o sentimento de autonomia e de reconhecimento (Dejours, 2004; Rossi, 2011). Por outro lado, pode ser fonte de pressões, dificuldades e desafios que podem causar sofrimento (Lancman \& Sznelwar, 2004; Rossi, 2011). Assim, a compreensão psicodinâmica do trabalho torna-se um instrumento importante para indicar quais elementos devem ser alvo de suporte, por parte da organização, no intuito de propiciar um ambiente de trabalho mais prazeroso e com menor possibilidade de potencializar o sofrimento dos trabalhadores (Carmo et al., 2017; Mendes, 2007; Oleto et al., 2013; Prestes, et al. 2010).

Neste sentido, a organização do trabalho tem um papel pri- mordial na mediação das vivências de prazer e sofrimento no trabalho. Dejours (1992) classifica a organização do trabalho em duas esferas: a primeira é conhecida como divisão do trabalho e, a outra, divisão dos homens (Mendes \& Facas, 2011). A divisão do trabalho é definida pela organização das tarefas, processos prescritos e modo de produção (Mendes \& Facas, 2011). A divisão dos homens compreende as responsabilidades relacionadas ao trabalho, as relações de poder, sistemas de hierarquias e de comandos, grau de autonomia nas atividades, e as possibilidades de cooperação e comunicação (Mendes \& Facas, 2011).

A organização do trabalho é notada como uma realidade social, na medida em que mobiliza e também é mobilizada pelo trabalhador que, por sua vez, investe sua subjetividade e constitui a intersubjetividade no trabalho (Andrade, Moraes, Tosoli, \& Wachelke, 2015; Mendes, \& Facas, 2011). É possível depreender, então, que existem forças visíveis e invisíveis, objetivas e subjetivas, psíquicas, sociais, políticas e econômicas que influenciam no ambiente de trabalho. Por conseguinte, essas forças podem vir, ou não, a deteriorar o ambiente organizacional, movimentando-o para um lugar de prazer ou sofrimento (Andrade et al., 2015; Mendes, 2007).

Ao encontro das discussões sobre a psicodinâmica dos aspectos subjetivos negativos e/ou positivos evocados pelo trabalho, está o suporte organizacional (SO), fator que também pode contribuir para a dinâmica entre prazer e sofrimento (Estivalete, Andrade, Faller, Stefanan, \& Souza, 2016). Em linhas gerais, o conceito de SO está associado às ações ofertadas pela organização que tendem a desenvolver seus trabalhadores, prover recursos, garantir autonomia, visibilidade e reconhecimento (Aselage \& Eisenberger, 2003; Padovan, 2005).

A percepção de $\mathrm{SO}$ envolve a crença do trabalhador sobre o quanto a organização valoriza as suas contribuições e cuida do seu bem-estar (Eisenberger, Huntington, Hutchison, \& Sowa, 1986). Relaciona-se, ainda, com a avaliação que os trabalhadores fazem sobre o fato da organização apoiar suas necessidades sócio emocionais, na medida em que recompensa e premia seus esforços (Allen, Armstrong, Reid, \& Riemenschneider, 2008).

Em outras palavras, a percepção de SO está diretamente associada às questões interacionais entre o trabalhador e a organização (Cardoso \& Baptista, 2015). Essa interação, quando percebida de forma positiva pelo trabalhador, indica que o local de trabalho é avaliado como agradável, configurando um espaço em que é possível aliar o desempenho ao bom relacionamento entre as pessoas e ao prazer no trabalho. Contudo, quando a visão do ambiente é negativa, as pessoas tornam-se mais suscetíveis a fatores psicossociais de sofrimento e adoecimento, como é o caso do estresse (Eisenberger et al., 1986).

Há consenso na literatura de que para a percepção de SO, algumas categorias devam ser analisadas, como os antecedentes e consequentes. As percepções de suporte organizacional dependem da relação e da troca entre superiores hierárquicos e subordinados. Sendo assim, a valorização e o reconhecimento das contribuições atuam como antecedentes da percepção positiva do ambiente de trabalho. Outro antecedente refere-se ao sentimento de estar sendo retribuído, fator composto por pagamentos, promoções, estabilidade no emprego, autonomia e treinamento. A justiça de procedimentos na distribuição de recursos para o melhor desempenho nas atividades, ou seja, a forma como o supervisor ou gestor contribui para a evolução dos trabalhadores, também são considerados antecedentes da percepção de SO (Eisenberger et al., 1986; Siqueira \& Gomide Jr., 2004).

A percepção de SO é considerada como um fator preponderante na contribuição para o bem-estar, comprometimento, motivação, qualidade de vida e felicidade no trabalho (Campos \& Estivalete, 2013; Estivalete et al., 2016; Paschoal, Torres, \& Porto, 2010). 
Assim, acrescentam-se como consequentes da percepção de SO, a diminuição das faltas no trabalho, menor intenção desligamento da empresa, aumento do desempenho, maior comprometimento afetivo com a organização e com a equipe de trabalho e maior cidadania no trabalho (Siqueira \& Gomide Jr., 2004). De forma direta ou indireta, a obtenção de percepção positiva de SO nutre as necessidades dos trabalhadores em nível emocional, instrumental e informacional (Campos \& Estivalete, 2013; Eisenberger et al., 1986).

Achados de pesquisas brasileiras e internacionais relacionaram o SO com as variáveis estresse, burnout, depressão e ansiedade (Bertoncello \& Borges-Andrade, 2015; Cardoso \& Baptista, 2015; Scott, Zagenczyk, Schippers, Purvis, \& Cruz, 2014). Apesar da constatação de que o SO pode ser um fator importante para a promoção de saúde no trabalho, nota-se carência de estudos que investiguem sua relação com as vivências de prazer e sofrimento no trabalho. É importante que se possa abordar as perspectivas intersubjetivas no trabalho hospitalar, de modo ampliado, ou seja, considerando-se tanto os profissionais com formação na área da saúde, quanto àqueles do corpo técnico-administrativo. Soma-se a isso, a justificativa de que todos estão sob o mesmo regime de organização do trabalho, demandando atenção e suporte (Malik, 2012).

A partir dos argumentos supracitados, que incluem a importância da análise da relação entre suporte organizacional e vivências de prazer e sofrimento no trabalho, considerando-se o conjunto de trabalhadores de um contexto hospitalar, foram analisadas as seguintes hipóteses: Hipótese (H1a) - A percepção de $\mathrm{SO}$ afeta a forma como o indivíduo percebe vivências de prazer no trabalho em um contexto hospitalar; Hipótese (H1b) - A percepção de SO afeta a forma como o indivíduo percebe vivências de sofrimento no trabalho em um contexto hospitalar.

\section{Método}

A pesquisa é correlacional, de corte transversal e recorreu à análise de variância e regressão linear para explicar a relação entre as variáveis do estudo. Estudos de coorte transversal observam a situação de um grupo em um determinado momento e assim são identificados dentro de uma população os desfechos existentes, podendo elencar fatores que podem ou não estar associados a estes em diferentes níveis de associação (Aragão, 2013).

\section{Participantes}

Participaram deste estudo 172 trabalhadores (enfermeiros e corpo técnico-administrativo) de um hospital filantrópico localizado no norte do Rio Grande do Sul. A instituição possui 934 empregados. É válido ressaltar que neste estudo não foram incluídos os médicos, em razão de que muitos deles atuam sem vínculo empregatício com a instituição. Desta forma, a homogeneidade da amostra poderia ficar comprometida.

A escolha da instituição hospitalar se deu por conveniência dos pesquisadores e a população foi escolhida de forma não probabilística. Os critérios de participação no estudo foram: ser trabalhador regular do hospital e ter idade entre 18 e 60 anos. Foram excluídos da pesquisa todos os indivíduos que eram trabalhadores terceirizados. As profissões ou cargos ocupados pelos participantes não foram utilizados como critério de inclusão ou exclusão nesse estudo, visto que não se buscou estudar uma área de atuação específica, mas sim, o profissional que está inserido na organização.

\section{Instrumentos}

Para coleta dos dados foi elaborado um questionário com três partes: 1) Escala de Percepção de Suporte Or- ganizacional (EPSO); 2) Escala de Indicadores de Prazer e Sofrimento no Trabalho (EIPST) e 3) Perguntas para elaboração do perfil sociodemográfico dos participantes.

A EPSO, utilizada para medir a Percepção de Suporte Organizacional, construída e validada no Brasil (Oliveira-Castro, Pilati e Borges-Andrade, 1999), foi baseada na proposta teórica de Eisenberger et al. (1986). Esse instrumento avalia quatro fatores. O primeiro deles é a gestão de desempenho abrangendo questões do tipo "valoriza novas ideias". O segundo fator denominado de carga de trabalho com questões do tipo "submete o trabalhador a uma carga excessiva de trabalho". O terceiro fator, suporte material, refere-se a afirmativas do tipo "Procura adquirir equipamentos modernos de trabalho". Por último, o fator denominado práticas organizacionais de ascensão, promoção e salários, constava de itens como "oferece raras oportunidades de promoção". A escala utilizada é do tipo Likert com respostas numa escala de 1 a 5 , que variam de "Discordo totalmente a Concordo Totalmente". Esta escala, no estudo de Oliveira-Castro et al. (1999), apresentou elevado índice de consistência interna $(\alpha=0,95)$ e explicação de $28 \%$ da variância das respostas da amostra aos itens.

A EIPST foi utilizada para medir o prazer e sofrimento no trabalho. Trata-se de uma escala que integra o Inventário de Trabalho e Riscos de Adoecimento (ITRA), que objetiva avaliar, a partir do modelo teórico da Psicodinâmica do Trabalho, o trabalho e os riscos de adoecimento por ele provocado, no que diz respeito a representações do contexto de trabalho, exigências (físicas, cognitivas e afetivas), vivências e danos. O ITRA é composto por quatro escalas independentes. Considerando-se a literatura sobre SO e contexto hospitalar, optou-se exclusivamente pela EIPST para garantir maior profundidade analítica com relação a este aspecto específico (Mendes \& Ferreira, 2007).

A EIPST é composta por quatro fatores: dois avaliam o prazer, realização profissional e liberdade de expressão. Os outros dois avaliam os fatores de sofrimento, falta de reconhecimento e esgotamento emocional. $\mathrm{O}$ instrumento foi criado e validado, inicialmente, por Ferreira e Mendes (2003) e revalidado após algumas adaptações (Mendes \& Ferreira, 2007). Constitui-se de uma escala do tipo Likert de sete pontos, que solicita ao respondente que avalie a ocorrência de vivências de prazer e sofrimento, nos últimos seis meses. Para classificar as médias de prazer e sofrimento, os resultados seguiram os parâmetros interpretativos do instrumento, segundo Mendes e Ferreira (2003). Para a dimensão vivências de prazer, médias maiores de 3,0 representam uma avaliação positiva e satisfatória do trabalho. Para as vivências de sofrimento, as médias maiores de 3,0 representam avaliação negativa-grave do ambiente de trabalho.

O questionário sociodemográfico foi elaborado especificamente para esta pesquisa, contendo perguntas sobre sexo, idade, escolaridade e tempo de vínculo na instituição hospitalar.

Para análise dos dados, o estudo seguiu quatro etapas: 1) análise de frequência para apresentar o perfil da amostra; 2) análise descritiva para demonstrar as médias das variáveis pertencentes a cada um dos fatores; 3) testes de confiabilidade (Alfa de Cronbach e Análise Fatorial Exploratória) para aferir a consistência das escalas e 4) ANOVA e Regressão linear para testar diferenças entre grupos e as hipóteses propostas no estudo. Importante destacar que apesar das escalas utilizarem pontuação Likert diferente, a análise dos dados ponderou os escores para evitar distorção entre as escalas de cinco pontos (Escala de SO) e de sete pontos (Escala de Vivências de Prazer e Sofrimento no Trabalho).

A escolha das variáveis para compor o modelo de análise seguiu os critérios estabelecidos no referencial teórico que sustenta as hipóteses propostas. Dessa forma, a variável independente SO contempla os fatores disponibilizados ao conjun- 
to dos trabalhadores que conformam o contexto hospitalar, sejam eles de setores de suporte técnico-administrativo, ou de atenção aos pacientes. Essa definição é fundamental para entender a organização hospitalar como um todo, reportando aos aspectos organizacionais que influenciam na variável dependente estabelecida, que são as vivências de prazer e sofrimento.

\section{Procedimentos de Coleta de Dados e Cuidados Éticos}

Esta pesquisa constituiu-se de um desdobramento de um projeto amplo, intitulado "Relação entre os riscos psicossociais, motivação, comprometimento no trabalho e suporte organizacional em um contexto hospitalar", o qual foi submetido à análise de Comitê de Ética em Pesquisa (CEP) aprovado sob número CAAE averiguar se os dados eram adequados para serem submetidos ao processo de análise de regressão (Pestana \& Gageiro, 2005). Para tanto, foi utilizado o critério de Kaiser- Meyer-Olkin (KMO) e de Esfericidade de Bartlett (> 0,5) (Dziuban \& Shirkey, 1974; Pestana \& Gageiro, 2005). Por fim, foi efetuada uma ANOVA para analisar se há diferença entre grupos e uma regressão linear múltipla com intuito de testar as hipóteses do estudo (Hair Jr. et al., 2005) e explicar a capacidade do modelo teórico, medido pelo coeficiente de determinação $\left(\mathrm{r}^{2}\right)$.

\section{Resultados}

Por meio de análises de frequências, conheceu-se o perfil sociodemográfico da amostra (Tabela 1)

Tabela 1

Dados sociodemográficos dos participantes

\begin{tabular}{|c|c|c|}
\hline Características sociográficas & $N$ & $\%$ \\
\hline \multicolumn{3}{|l|}{ Sexo } \\
\hline Masculino & 29 & 16,9 \\
\hline Feminino & 143 & 83,1 \\
\hline \multicolumn{3}{|l|}{ Faixa etária (anos) } \\
\hline $18-25$ & 34 & 19,4 \\
\hline $26-35$ & 78 & 45,4 \\
\hline $36-50$ & 37 & 21,7 \\
\hline $51-60$ & 23 & 13,5 \\
\hline \multicolumn{3}{|l|}{ Escolaridade } \\
\hline Ensino Médio completo & 91 & 52,9 \\
\hline Superior completo & 54 & 31,4 \\
\hline Pós-graduação & 27 & 15,7 \\
\hline \multicolumn{3}{|l|}{ Tempo de vínculo } \\
\hline Entre 1 e 3 anos & 86 & 50,0 \\
\hline Entre 4 e 10 anos & 60 & 34,9 \\
\hline Entre 11 e 20 anos & 20 & 11,7 \\
\hline Mais de 21 anos & 6 & 3,34 \\
\hline \multicolumn{3}{|l|}{ Setores de atuação } \\
\hline Saúde & 88 & 51,2 \\
\hline Administração & 70 & 40,7 \\
\hline Infraestrutura/Manutenção & 14 & 8,1 \\
\hline \multicolumn{3}{|l|}{ Composição do setor Saúde (51,2\%) } \\
\hline Enfermeiros & 29 & 31,9 \\
\hline Técnicos de enfermagem & 51 & 58,2 \\
\hline Nutricionistas, Fisioterapeutas, Psicólogos e A. Sociais & 8 & 9,9 \\
\hline
\end{tabular}

Nota. Fonte: elaboração dos autores (2020).

57331416.2.0000.5319. Os dados foram coletados nas dependências do hospital entre os meses de agosto e setembro de 2017.

Os entrevistadores coletaram os dados em um espaço reservado especificamente para tal, de modo a resguardar a privacidade e o sigilo das respostas. Os respondentes dirigiram-se à sala de acordo com a disponibilidade de tempo, sem descumprir suas obrigações laborais.

\section{Procedimentos de Análise de Dados}

Os dados coletados foram analisados por meio do software estatístico Statistical Package for the Social Sciences $($ SPSS), versão 20 para $W$ indows ${ }^{\circledR}$. A primeira análise foi referente à confiabilidade das escalas utilizadas, por meio dos coeficientes de Alpha de Cronbach (Hair Jr., Joseph, Babin, Money, \& Samouel, 2005). Na segunda etapa, foram realizadas as análises descritivas, por meio de cálculos de frequência e médias. Na sequência, procurou-se
Posteriormente, foi realizada uma ANOVA com a variável setores de atuação, para evidenciar se havia diferenças entre os setores que atuam diretamente com os pacientes (trabalhadores com formação em saúde) e os trabalhadores do quadro técnico-administrativo, considerando-se os fatores vivências de prazer e sofrimento e SO. A análise não encontrou diferenças estatisticamente significativas entre as médias dos grupos.

Em seguida, foi realizado o teste de confiabilidade das escalas aplicadas. Em ambas as escalas foi constatado um bom nível de confiabilidade, medido pelo Alfa de Cronbach $(\alpha)$, estabelecido acima do mínimo recomendado de 0,70 (Hair Jr. et al., 2005). O alfa da Escala de Indicadores de Prazer e Sofrimento no Trabalho foi de 0,914 e o da Escala de Suporte Organizacional foi de 0,792.

Também foi realizada Análise Fatorial Exploratória (AFE) das escalas utilizadas para preparar o teste das hipóteses. Foram observados os pontos críticos de cada índice, especialmente os eigenvalues, pontos de sedimentação relativos a cada um dos fatores 
e as comunalidades (Figueiredo Filho \& Silva Júnior, 2010). A Tabela 2 apresenta alguns escores importantes para atestar a fatorabilidade da amostra, considerando-se que o ponto crítico do teste de Kaiser-Meyer-Olkin (KMO) é 0,60 e do teste de Esfericidade de Bartlett deve ser significativo $(p<0,05)$ (Dziuban \& Shirkey, 1974; Hutcheson \& Sofroniou, 1999; Pestana \& Gageiro, 2005).

Tabela 2

Resultado da AFE das escalas

\begin{tabular}{ccccc}
\hline Escala & N Fatores & KMO & $\begin{array}{c}\text { Variância } \\
\text { extraída }\end{array}$ & Sig. \\
\hline $\begin{array}{c}\text { Vivências de Prazer e } \\
\text { Sofrimento } \\
\text { Suporte no Trabalho }\end{array}$ & 4 & 0,899 & 60,587 & 0,000 \\
\hline
\end{tabular}

Nota. Fonte: Autores (2020)

Posteriormente, foi realizada a análise descritiva para destacar as médias das respostas de cada uma das variáveis do estudo. Para o constructo "vivências de prazer", observou-se que as médias variaram entre 3,29 e 4,59. As duas variáveis com as maiores médias de vivências de prazer foram "Orgulho pelo que faço" e "Identificação com as minhas tarefas", enquanto as duas com menores pontuações foram "Reconhecimento" e "Valorização".

No que se refere às vivências de sofrimento, as médias estabeleceram-se entre 1,81 e 3,04, indicando escores entre baixo e moderado de sofrimento no trabalho. As perguntas com maiores médias foram "estresse", "sobrecarga e esgotamento" e "esgotamento emocional", resultando em índices moderado-críticos. As menores médias foram percebidas em relação à "discriminação" e "inutilidade" (Tabela 3)

Tabela 3

Distribuição da média e desvio-padrão dos fatores

\begin{tabular}{cccc}
\hline Vivências & Variável & Média & DP \\
\hline \multirow{3}{*}{ Prazer } & Orgulho pelo que faço & 4,59 & 0,74 \\
& Identificação com as minhas tarefas & 4,21 & 0,82 \\
& Reconhecimento & 3,29 & 1,14 \\
& Valorização & 3,36 & 1,15 \\
\hline \multirow{3}{*}{ Sofrimento } & Estresse & 3,04 & 1,02 \\
& Sobrecarga & 3,04 & 1,17 \\
& Esgotamento emocional & 2,91 & 1,02 \\
& Discriminação & 1,81 & 1,01 \\
& Inutilidade & 1,88 & 0,95 \\
\hline
\end{tabular}

Nota. Fonte: Autores (2020)

Ao analisar as médias descritivas de $\mathrm{SO}$, obteve-se números que variaram entre 1,41 e 4,20, nos quais as afirmativas com menores médias foram "Obriga o trabalhador a cumprir horas extras" $(1,41 ; \sigma, 73)$, "Suga a energia do trabalhador" (2,14; $\sigma 1,62)$. As maiores médias foram das afirmativas relacionadas à percepção de suporte material, sendo elas: "Fornece materiais em quantidade suficiente" $(4,20 ; \sigma 0,85)$ e "Fornece os equipamentos necessários ao desempenho eficaz" (3,98; $\sigma$ 1,05).

Para testar as hipóteses desse estudo, foi realizada a análise de regressão linear, sendo definido o SO como variável independente e as vivências de prazer e sofrimento como variável dependente. A primeira hipótese testou a influência da percepção de SO nas vivências de prazer (H1a). Os resultados apontaram que as vivências de prazer no trabalho são influenciadas de forma positiva e significativa por dois fatores do SO: "Suporte de Gestão de Desempenho" $(\beta$ 0,527; $p<0,001)$ e o "Suporte Material" $(\beta, 301 ; p<0,001)$. A regressão constatou uma boa capacidade explicativa do modelo $\left(r^{2}=0,579\right)$.

Em seguida, foi testada a influência da percepção de SO nas vivências de sofrimento no trabalho (H1b). A regressão confir- mou um efeito significativo e negativo do SO sobre as vivências, com uma boa capacidade explicativa do modelo $\left(r^{2}=0,461\right)$. Os fatores com maior significância negativa sobre as vivências de sofrimento foram "Suporte carga de trabalho" $(\beta-6,486 ; p<0,001)$ e "Suporte na Gestão do Desempenho" $(\beta-2,044 ; p=0,043)$.

\section{Discussão}

O trabalho envolve a subjetividade de cada sujeito, podendo ser fonte de sofrimento e de fadiga para uns e de prazer para outros (Carmo et al., 2017; Dejours, 2008). Não se pode afirmar, de forma apriorística, que o trabalho é equilibrante ou fatigante, prazeroso ou de sofrimento. É apropriado apenas apontar quais aspectos do trabalho que são positivos e negativos (Santos \& Pires, 2007).

Desse modo, as primeiras análises realizadas neste estudo apontaram os aspectos positivos e negativos, expressos em termos de vivências de prazer e sofrimento que o trabalho dentro de um hospital pode significar. Para os trabalhadores pesquisados, o prazer no trabalho é sinônimo de sentimento de gratificação, sendo descrito por níveis elevados de orgulho e identificação com as tarefas. Segundo Rosado, Russo \& Maia (2015), Malik (2012), Santos \& Pires (2007), e Sangy (2017) a natureza humanística, social e de promoção à vida no trabalho em hospitais influencia a forma como o trabalhador sente a função que exerce, mantendo sentimentos positivos e de bem-estar, independentemente das diferentes áreas de atuação.

Por outro lado, as vivências de sofrimento para esta amostra estão ligadas ao estresse, sobrecarga no trabalho e esgotamento emocional. Esses resultados vão ao encontro do estudo realizado Rosado et al. (2015) que compreendem o trabalho dentro dos hospitais como complexo, existindo predominantemente um desgaste físico e emocional atrelado a ele, sendo recorrente que os profissionais se sintam sobrecarregados com as tarefas, elevando o nível de estresse. O estudo realizado por Sangy (2017) com profissionais de um hospital incluindo trabalhadores do corpo clínico, enfermagem, administração e conservação, também identificou alguns fatores que interferem na saúde física e mental dos trabalhadores, tais como: estresse e pressão, lidar com a morte, violência e vulnerabilidade, conflitos relacionais internos e com pacientes.

Nas análises descritivas foi possível verificar que os trabalhadores da instituição hospitalar estão satisfeitos com o suporte material que a organização oferece. Este mesmo resultado foi encontrado no estudo de Ludwig (2015), onde o suporte material igualmente se apresentou com médias elevadas, representando que ter percepção de suporte material é trabalhar em um ambiente que oferece máquinas, equipamentos, ou seja, é um ambiente adequado de trabalho. Além disso, ter percepção de suporte material significa que a organização proporciona aos trabalhadores as condições físicas de trabalho e materiais de qualidade, independentemente do desempenho individual de cada sujeito, de modo que esses fatores podem exercer influência direta no sentimento de bem-estar (Paschoal et al., 2010).

Nas hipóteses H1a e H1b, foram testadas as relações do SO nas vivências de prazer e sofrimento no trabalho. Além da alta capacidade explicativa do modelo, que foi de 58\% para as vivências de prazer e $46 \%$ para vivências de sofrimento, a análise mostrou uma relação altamente significativa entre as vivências e o SO, mostrando que os indivíduos, quando percebem suporte, vivenciam sentimentos de prazer no trabalho, principalmente quando recebem suporte na gestão de desempenho e suporte material. Esse resultado evidencia que a variável SO é promissora para a compreensão de experiências subjetivas positivas e/ou negativas no trabalho, indo ao encontro das pesquisas realizadas por Campos e Estivalete, (2013), Estivalete et al. (2016), Pascho- 
al et al. (2010). Trata-se de uma variável que, quando percebida pelos trabalhadores, está intimamente ligada aos aspectos positivos no trabalho, no sentimento de bem-estar e na felicidade do trabalhador, como encontrado em Estivalete et al. (2016).

No contexto hospitalar, um bom suporte organizacional para os trabalhadores passa pelo suporte material e apoio na gestão do desempenho. As condições de trabalho devem ser vistas como adequadas incluindo desde o ambiente, o pessoal, até o material necessário para o desempenho das atividades. Além disso, a percepção que os trabalhadores têm sobre o ambiente hospitalar estão ligadas à segurança do doente, sendo que este acaba por gerar nos trabalhadores um sentimento de obrigação pelo seu desempenho nas atividades, e consequentemente pode vir a gerar sentimentos negativos de esgotamento, caso o suporte não seja percebido (Cordes \& Dougherty, 1993; Cruz, 2015).

Uma organização de sucesso constitui-se de trabalhadores com um bom nível de percepção de suporte organizacional, pois pessoas que se sentem apoiadas são mais felizes na vida pessoal e profissional, se comprometem com a organização e oferecem o seu melhor para as pessoas que procuram o serviço. No caso de hospitais, profissionais que se sentem apoiados, oferecem o tratamento adequado, com qualidade e segurança necessária (Cruz, 2015).

A ausência de SO nas questões direcionadas à sobrecarga de trabalho, contribui para ocorrência de vivências de sofrimento. Esse resultado mostra-se importante na medida em que organizações consomem as energias de seus trabalhadores, submetendo-os a cargas excessivas de trabalho, e não oferecendo o suporte adequado, é provável, que os trabalhadores passem a vivenciar sofrimento, tendo possíveis riscos de adoecimento (Estivalete et al., 2016; Paschoal et al., 2010).

Paschoal et al. (2010) evidencia que quanto menor a percepção de suporte para as sobrecargas no trabalho, maior será o afeto negativo pelo trabalho, podendo causar níveis elevados de estresse, corroborando os achados dessa pesquisa. Acrescenta-se a esta compreensão, o fato de que as ações organizacionais, quando geram demandas excessivas para o trabalhador, acarretam em intensificação de vivências de sofrimento (Brunetto, Stephen, Farr-Wharton, Shacklock, \& Shriberg, 2017; Paschoal et al., 2010).

Segundo Wu, Ge, Sun, Wang \& Wang (2011), os agentes causadores de transtornos de humor entre os trabalhadores são a sobrecarga e a falta de apoio do local de trabalho. No Brasil, os transtornos de humor causados pelo trabalho correspondem a $14 \%$ dos afastamentos anuais, prejudicando não apenas a saúde dos trabalhadores, mas também o bem-estar dos familiares (Organização Internacional do Trabalho [OIT], 2016).

A percepção de suporte organizacional, em relação à dimensão "gestão do desempenho" demonstrou relação positiva com as vivências de prazer no trabalho. Do mesmo modo, obteve resultados negativos em relação às vivências de sofrimento no trabalho, atrelados ao sentimento de não consideração das ideias, falta de valorização do profissional, de capacitação e acesso a informações. Achados como estes corroboram os estudos de Neves (2012) e Scott et al. (2014), enfatizando que a precária percepção de SO na gestão do desempenho influencia no comprometimento dos trabalhadores com a organização, além de aumentar os níveis de sentimentos negativos com o trabalho. $\mathrm{O}$ trabalhador passa a se sentir estressado e não valorizado, e em longo prazo, pode vir a acarretar no desenvolvimento de patologias, físicas e psicológicas, além do absenteísmo, e sintomas de depressão (Hyeda \& Maluf, 2017; Neves, 2012; Scott et al., 2014).

Como resultado principal, a pesquisa confirmou as hipóteses de que as vivências de prazer e sofrimento no trabalho são afetadas pela forma como os trabalhadores hospitalares percebem o suporte organizacional, assim como no estudo de Bertoncello et al. (2017). Não foram encontradas diferenças estatisticamente significativas, em referência à hipótese, entre o grupo de trabalhadores da área da saúde e os dos setores administrativos e de apoio. Isso permite inferir que, para a efetividade do serviço hospitalar, o corpo de trabalhadores deve ser percebido em amplitude e multidisciplinaridade, a partir das práticas de gestão e de SO, não parecendo ser adequado negligenciar uma área em detrimento da outra.

\section{Considerações Finais}

O estudo procurou identificar a influência da percepção de SO sobre as vivências de prazer e sofrimento no trabalho. $\mathrm{O}$ principal achado da pesquisa se refere à identificação de que o SO, manifesto pelo suporte material e apoio para um melhor desempenho, propicia aos trabalhadores vivências de prazer no trabalho, enquanto a falta de suporte, quando relacionada à sobrecarga de trabalho e má gestão do desempenho, gera vivências de sofrimento.

Os resultados mostram a importância da percepção de suporte no âmbito organizacional, pressupondo que a organização valoriza suas contribuições e cuida do seu bem-estar. Resultados também defendidos por Eisenberger et al. (1986), Oliveira-Castro et al. (1999), e Estivalete et al. (2016), demonstrando que o suporte no trabalho pode atuar de forma direta ou indireta na construção de um ambiente saudável de trabalho, visto que ele supre as necessidades dos trabalhadores em nível emocional, instrumental e informacional; esses fatores são preditores dos afetos positivos vivenciados no trabalho.

Com base no exposto, a pesquisa cumpriu seu objetivo principal, discutindo a importância do SO como preditor das vivências de prazer e sofrimento para os profissionais em um contexto hospitalar. A singularidade deste estudo reside na inclusão de profissionais que atuam tanto nas áreas administrativas, quanto às ligadas diretamente ao cuidado médico. Assim, a investigação pode contribuir com os estudos científicos nacionais, possibilitando a discussão da saúde mental do trabalhador em espaços de trabalho menos visíveis no âmbito hospitalar.

Entre as limitações deste estudo, é importante considerar que no decurso da coleta de dados, o hospital estava passando por um processo de acreditação, momento em que a instituição passou a impor novas exigências aos trabalhadores no que se refere às mudanças comportamentais. Embora não seja possível mensurar o efeito deste "evento" na percepção dos trabalhadores durante a coleta, sabe-se que a instauração de uma política de metas e avaliação de desempenho podem influenciar na percepção de $\mathrm{SO}$ e esse fator pode ter influenciado nas opiniões sobre o contexto de trabalho. Os resultados encontrados mostram uma relação tanto positiva quanto negativa do SO com a gestão do desempenho, evidenciando que o tema da gestão do desempenho está na ordem do dia, neste momento histórico do Hospital.

Reitera-se que o modelo de análise incorporou trabalhadores em uma amostra homogênea, qual seja, trabalhadores de uma instituição hospitalar que conformam setores complementares para oferta de cuidados em saúde. Entretanto, este modelo pode apresentar falhas por um possível desiquilíbrio entre participantes e setores para uma amostra mais significativa. Em outras palavras, embora a taxa de resposta dos questionários tenha sido satisfatória, a falta de critérios para definição do cálculo amostral podem ser objeto de pesquisas futuras. Importante reiterar que o estudo foi realizado em uma única organização, escolhida por conveniência. Assim, não foram realizados cálculos amostrais ou segmentação da amostra de forma a orientar para uma representatividade dos setores que compõem a instituição analisada. Estas escolhas indicam vieses de seleção que podem ser testados em estudos futuros, segmentando a amostra por setores de maneira 
mais criteriosa e ampliando a análise para outras instituições para investigar e comparar os achados deste estudo a outros contextos hospitalares. Por outro lado, a confirmação dos achados desta pesquisa em outros estudos já publicados ratifica a relação entre os fatores de desempenho e a exigência de um SO adequado, independente do momento da organização ou contexto analisado.

Entre as lacunas deste estudo está a dificuldade em generalizar conclusões em razão da especificidade do contexto hospitalar analisado. Assim, sugere-se que a pesquisa seja replicada em outras instituições hospitalares, sendo elas públicas ou privadas, ampliando o número da amostra e consequentemente expandindo a investigação sobre a temática. Para além disso, incentivam-se que outros estudos em contexto hospitalar incorporem os trabalhadores administrativos e de serviços de apoio.

Por fim, acredita-se que os resultados desta pesquisa permitem dar visibilidade aos aspectos subjetivos do trabalho, possibilitando a discussão da saúde mental do trabalhador em áreas menos discutidas no contexto hospitalar. Igualmente, sugeriram indicativos de como construir um ambiente saudável de trabalho, permitindo subsidiar a realização e ampliação de ações preventivas e assistenciais direcionadas para os trabalhadores, que fortaleçam o seu crescimento individual, fornecendo boas condições de trabalho, com mais saúde, dignidade e reconhecimento. Além disso, pode-se dizer que o SO pode ser o ponto de partida para novas estratégias de gestão, e, consequentemente, um fator que influencia em sentimentos positivos diante do trabalho.

\section{Referências}

Allen, M. W., Armstrong, D. J., Reid, M. F., \& Riemenschneider, C. K. (2008). Fatores que afetam o suporte organizacional percebido dos funcionários de TI. Informação e Gestão , 45(8), 556-563. https://doi.org/10.1016/i. im.2008.09.003

Aselage, J., \& Eisenberger, R. (2003). Perceived organizational support and psychological contracts: A theoretical integration. Journal of Organizational Behavior: The International Journal of Industrial, Occupational and Organizational Psychology and Behavior, 24(5), 491-509. https://doi.org/10.1002/job.211

Andrade, A. L. D., Moraes, T. D., Tosoli, A. M., \& Wachelke, J. (2015). Burnout, clima de segurança e condições de trabalho em profissionais hospitalares. Revista Psicologia Organizações e Trabalho, 15(3), 233-245. https://doi. org/10.17652/rpot/2015.3.565

Aragão, J. (2013). Introdução aos estudos quantitativos utilizados em pesquisas científicas. Revista Práxis, 3(6), 59-62. https://doi.org/10.25119/ praxis-3-6-566

Bendassolli, P. F. (2007). Felicidade e trabalho. GV -executivo, 6(4), 57-61.

Bendassolli, P. F. (2012). Saúde e trabalho podem caminhar juntos?. GV-executivo, 11(2), 26-30

Bertoncello, B., \& Borges-Andrade, J. E. (2015). Relações entre Suporte Organizacional e Saúde Mental do Trabalhador. R. Laborativa, 4(2), 85-102.

Bertoncello, B., Sousa, E., Marques, D., \& Oliveira, E. C. (2017). Relações entre Estresse, Saúde Mental e Suporte Organizacional em um Hospital de Ensino. CLAIQ 2017, 2, 523-528.

Bueno, M., \& Macêdo, K. B. (2012). A Clínica psicodinâmica do trabalho: De Dejours às pesquisas brasileiras. ECOS-Estudos Contemporâneos da Subjetividade, 2(2), 306-318.

Brito, B. C., Santos, F. C., Soares, M. I., \& Camelo, S. H. H. (2014). Riscos psicossociais relacionados ao trabalho do enfermeiro hospitalar e estratégias de gerenciamento uma revisão integrativa. Gestão e Saúde, 6(1), 437-450. https://doi.org/10.18673/gs.v6i1.13746

Brunetto Y., Stephen, T.T., Farr-Wharton, R., Shacklock K., \& Shriberg, A. (2017). Individual and organizational support: Does it affect red tape, stress and work outcomes of police officers in the USA?, Personnel Revien, 46(4), 750-766.

Camelo, S. H. (2011). O trabalho em equipe na instituição hospitalar: uma revisão integrativa. Cogitare enfermagem, 16(4), 734-40.

Campos, S. A. P., \& Estivalete, V. D. F. B. (2013). O trabalho de quem ensina: suporte social, suporte organizacional e comprometimento. Revista Alcance (Online), 20(1), 58. https://doi.org/10.14210/alcance.v20n1.p058-078

Cardoso, H. F., \& Baptista, M. N. (2015). Escala de suporte laboral: correlação com suporte social, estresse e suporte organizacional. Revista Psicologia Organizações e Trabalho, 15(4), 374-383. https://doi.org/10.17652/ rpot/2015.4.631
Carmo, J. G. M., Guimarães, L. D. V. M., \& de Lima Caeiro, M. (2017). Prazer e sofrimento no trabalho: vivências de mulheres soldados da pmmg. FarolRevista de Estudos Organizacionais e Sociedade, 3(8), 1278-1322. https://doi. org/10.25113/farol.v3i8.3180

Cordes, C. L., \& Dougherty, T. W. (1993). A review and an integration of research on job burnout. Academy of management reviem, 18(4), 621-656.

Costa, M. T. P., Borges, L. D. O., \& Barros, S. C. (2015). Condições de trabalho e saúde psíquica: Um estudo em dois hospitais universitários. Revista Psicologia Organizaçoes e Trabalbo, 15(1), 43-58.

Cruz, A. M. R. T. (2015). Percepsão de suporte organizacional, engagement e segurança do doente (Dissertação de Mestrado em Gestão e Economia da Saúde, Universidade de Coimbra). Recuperado de https://estudogeral.sib.uc.pt/ handle/10316/29960

Dziuban, C. D. \& Shirkey, E. C. (1974). When is a correlation matrix appropriate for factor analysis? Some decision rules. Psychological Bulletin, 81(6), 358-361. https://doi.org/10.1037/h0036316

Dejours, C. (1999). Sofrimento, prazer e trabalho. Anais das Conferencias Brasileiras: identidade, reconhecimento e transgressão no trabalbo, São Paulo, SP, Brasil.

Dejours, C. (2005). O fator bumano. Rio de Janeiro, RJ: Editora da Fundação Getulio Vargas.

Dejours, C. (2008). Trabalho, tecnologia e organização: A avaliação do trabalho submetida à prova do real (1a ed.). São Paulo, SP: Blucher.

Dejours, C. (2015). A loucura do trabalho (6a ed.). São Paulo, SP: Cortez.

Eisenberger, R., Huntington, R., Hutchison, S., \& Sowa, D. (1986). Perceived Organizational Support. Journal of applied psychology, 71(3), 500-507. https:// doi.org/10.1037/0021-9010.71.3.500

Estivalete, V. D. F. B., de Andrade, T., Faller, L. P., Stefanan, A. A., \& Souza, D. L. (2016). Suporte social e suporte organizacional como antecedentes do bem-estar no trabalho: a perspectiva de colaboradores de uma empresa de logística ferrovia. Revista de Administração da Unimep, 14(2), 31-56. https:// doi.org/10.15600/1679-5350/rau.v14n2p31-56

Ferreira, M. C., \& Mendes, A. M. (2003). Trabalho e riscos de adoecimento: o caso dos auditores-fiscais da previdência social brasileira. Brasília: Ler, Pensar e Agir.

Figueiredo Filho, D. B., \& Silva Júnior, J. A. D. (2010). Visão além do alcance: uma introdução à análise fatorial. Opinião pública, 16(1), 160-185. https://doi. org/10.1590/S0104-62762010000100007

Fleury, O. L. F. de, Formiga, N. S., Souza, M. A. de, \& Souza, M. A. F. de. (2017). Escala de Percepção de Suporte Organizacional: evidência da estrutura fatorial em trabalhadores brasileiros. Revista Psicologia em Pesquisa, 11(1), 1-10. https://doi.org/10.24879/201700110010033

Flores, V. D. C., \& Moura, E. P. G. de. (2018). Significados de trabalho, prazer e sofrimento no ofício de agentes funerários. Revista Psicologia Organizaacōes e Trabalho, 18(1), 326-334. https://doi.org/10.17652/rpot/2018.1.13337

Freitas, L. G., Augusto, M. M., \& Mendes, A. M. (2014). Vivências de prazer e sofrimento no trabalho de profissionais de uma fundação pública de pesquisa. Psicologia em Revista, 20(1), 34-55. https://doi.org/10.5752/P.16789523.2014v20n1p34

Giongo, C. R., Monteiro, J. K., \& Sobrosa, G. M. R. (2015). Psicodinâmica do trabalho no Brasil: revisão sistemática da literatura. Temas em Psicologia, 23(4), 803-814. https://doi.org/10.9788/TP2015.4-01

Hair Jr., J., Joseph F., Babin, B., Money, A. H., \& Samouel, P. (2005). Fundamentos de métodos de pesquisa em administracão. Porto Alegre, RS: Bookman.

Hutcheson, G. D. \& Sofroniou, N. (1999). The multivariate social scientist: Introductory statistics using generalized linear models. London: Sage Publications.

Hyeda, A., \& Maluf, E. M. C. P. (2017). A relação entre o suporte organizacional no trabalho e o risco para doenças crônicas não transmissíveis em um serviço de saúde. Revista Brasileira de Medicina do Trabalbo, 15(2), 134-141. https://doi.org/10.5327/Z1679443520176046

Lancman, S., Sznelwar, L. I. (2004). Christophe Dejours: da psicopatologia à psicodinamica do trabalho. Rio de Janeiro, RJ:Editora Fiocruz:

Ludwig, N. C. (2015). Reconhecimento e percepcão de suporte organizacional: um estudo entre trabalhadores dos serviços compartilhados regional Norte e Nordeste da Petrobras (Dissertação de Mestrado em Administração, Universidade Federal da Bahia). Recuperado de http://www.adm.ufba.br/sites/default/files/ publicacao/arquivo/natalia costa ludwig.pdf

Malik, A. M. M. (2012). Trabalho em hospitais. Tempus Actas de Saúde Coletiva, 6(4), 63-76. https://doi.org/10.18569/tempus.v6i4.1205

Mendes, A. M. (2007). Psicodinâmica do trabalho: teoria, método e pesquisas. São Paulo, SP: Casa do Psicólogo.

Mendes, A. M., \& Facas, E. P. (2011). Subjetividade e trabalho com automação. Em R. D. de. Moraes \& A. C. L. Vasconcelos (Eds.), Subjetividade e trabalho com automação: Estudo piloto no polo industrial de Manaus (pp. 18-37). Manaus, AM: Editora da Universidade Federal do Amazonas.

Mendes, A. M., \& Ferreira, M. C. (2007). Inventário sobre Trabalho e Riscos de Adoecimento - ITRA: Instrumento auxiliar de diagnóstico de indicadores críticos no trabalho. Em A. M. Mendes (Ed.), Psicodinâmica do Trabalho: teoria, método e pesquisas (pp. 111-126). São Paulo: Casa do Psicólogo. 
Neves, V. F. (2012). Impacto da satisfação no trabalho e da percep̧̧ão de suporte organizacional sobre a Sindrome de Burnout em trabalhadores de enfermagem de um Hospital Universitário (Dissertação de Mestrado em Ciências Humanas, Universidade Federal de Uberlândia, Uberlândia). Recuperado de https:// repositorio.ufu.br/handle/123456789/17166

Nogueira, L. S,. Sousa, R. M. C. de, Guedes, E. S., Santos, M. A. dos, Turrini, R. N., Teresa, \& Cruz, D. A. L. M. da. (2018). Burnout and nursing work environment in public health institutions. Revista Brasileira de Enfermagem, 71(2), 336-342. https://doi.org/10.1590/0034-7167-2016-0524

Oleto, A. D. F., Melo, M. C. D. O. L., \& Lopes, A. L. M. (2013). Análise bibliométrica da produção sobre prazer e sofrimento no trabalho nos encontros da Associação Nacional de Pós-Graduação em Administração (2000-2010). Psicologia ciência e profissão, 33(1), 60-73. https://doi. org/10.1590/S1414-98932013000100006

Oliveira-Castro, G. A. D., Pilati, R., \& Borges-Andrade, J. E. (1999). Percepção de suporte organizacional: desenvolvimento e validação de um questionário. Revista de Administração Contemporânea, 3(2), 29-51. https://doi.org/10.1590/ $\underline{\text { S1415-65551999000200003 }}$

Organização Internacional do Trabalho (2016). Stress de trabalho: Um desafio coletivo. Recuperado de http://www.ilo.org/wcmsp5/groups/public/---ed protect/---protrav/---safework/documents/publication/wcms 466547.pdf

Paschoal, T., Torres, C. V., \& Porto, J. B. (2010). Felicidade no trabalho: relações com suporte organizacional e suporte social. RAC-Revista de Administração Contemporânea, 14(6), 1054-1072. https://doi.org/10.1590/S1415$\underline{65552010000700005}$

Penaforte, K. L., \& Araújo, S. T. (2016). Riscos psicossociais relacionados ao trabalho: percepção dos profissionais de enfermagem. Revista de enfermagem UFPE, 10(11), 3831-3839. https://doi.org/10.5205/reuol.9881-87554-1EDSM1011201603

Pestana, M. H., \& Gageiro, J. N. (2005). Descobrindo a regressão: com a complementaridade do SPSS. Lisboa: Edições Sílabo.

Prestes, F. C., Beck, C. L. C., da Silva, R. M., Tavares, J. P., Camponogara, S., \& Burg, G. (2010). Prazer-sofrimento dos trabalhadores de enfermagem de um serviço de hemodiálise. Revista Gaúcha de Enfermagem, 31(4), 738-745. https://doi.org/10.1590/S1983-14472010000400018

Sangy, M. (2017). Saúde no Trabalho: intervenção psicossocial com trabalhadores de um hospital público. Anais do II Congresso Interdisciplinar de Pesquisa, Iniciação Cientifica e Extensão. Belo Horizonte.

Santos, A. P. J., \& Pires, D. E. P. (2007). O trabalho em emergência: entre o prazer e o sofrimento. Revista Eletrônica de Enfermagem, 9(3), 617-629. https://doi. org $/ 10.5216 /$ ree.v9i3.7445

Scott, K. L., Zagenczyk, T. J., Schippers, M., Purvis, R. L., \& Cruz, K. S. (2014). Co-worker exclusion and employee outcomes: An investigation of the moderating roles of perceived organizational and social support. Journal of Management Studies, 51(8), 1235-1256. https://doi.org/10.1111/joms.12099

Šimunić, A., \& Gregov, L. (2012). Conflict between work and family roles and satisfaction among nurses in different shift systems in Croatia: a questionnaire survey. Archives of Industrial Hygiene and Toxicology, 63(2), 189197. https://doi.org/10.2478/10004-1254-63-2012-2159

Siqueira, M. M. M., \& Gomide Jr., S. (2004). Psicologia, organizações e trabalho no Brasil. Porto Alegre: Artmed.

Rodrigues, C. M. L., \& Faiad, C. (2019). Pesquisa sobre riscos psicossociais no trabalho: estudo bibliométrico da produção nacional de 2008 a 2017. Revista Psicologia Organizações e Trabalho, 19(1), 571-579. https://doi.org/10.17652/ rpot/2019.1.15424

Rosado, I. V. M., Russo G. H. A., \& Maia, E. M. (2015). Produzir saúde suscita adoecimento? As contradições do trabalho em hospitais públicos de urgência e emergência. Revista Ciência \& Saúde Coletiva, 20(10), 3021-3032. https://doi.org/10.1590/1413-812320152010.13202014

Rossi, E. Z. (2011). Método e pesquisa em Psicodinâmica do trabalho. Em A. M. Mendes, A. R. C. Merlo, C. F. Morrone, \& E. P. Facas. (Org.), Psicodinâmica e Clínica do trabalho: Temas interfaces e casos brasileiros (pp. 113-124). Curitiba, PR: Juruá.

Wu, H., Ge, C. X., Sun, W., Wang, J. N., \& Wang, L. (2011). Depressive symptoms and occupational stress among Chinese female nurses: the mediating effects of social support and rational coping. Res Nurs Health, 34(5), 401-407. https://doi.org/10.1002/nur.20449

\section{Informações sobre os autores:}

\section{Manueli Tomasi}

Av. Júlio de Castilhos, 450, Caxias do Sul (RS), Brasil.

E-mail: manuelitomasi5@gmail.com

\section{Vanessa Rissi}

E-mail: vanessa.rissi@imed.edu.br

\section{Jandir Pauli}

E-mail: jandir.pauli@imed.edu.br 